\title{
Running coupling: Does the coupling between dark energy and dark matter change sign during the cosmological evolution?
}

\author{
Yun-He $\mathrm{Li}^{1}$ and Xin Zhang ${ }^{1,2, *}$ \\ ${ }^{1}$ Department of Physics, College of Sciences, Northeastern University, Shenyang 110819, China \\ ${ }^{2}$ Center for High Energy Physics, Peking University, Beijing 100080, China
}

\begin{abstract}
In this paper we put forward a running coupling scenario for describing the interaction between dark energy and dark matter. The dark sector interaction in our scenario is free of the assumption that the interaction term $Q$ is proportional to the Hubble expansion rate and the energy densities of dark sectors. We only use a time-variable coupling $b(a)$ (with $a$ the scale factor of the universe) to characterize the interaction $Q$. We propose a parametrization form for the running coupling $b(a)=b_{0} a+b_{e}(1-a)$ in which the early-time coupling is given by a constant $b_{e}$, while today the coupling is given by another constant, $b_{0}$. For investigating the feature of the running coupling, we employ three dark energy models, namely, the cosmological constant model $(w=-1)$, the constant $w$ model $\left(w=w_{0}\right)$, and the time-dependent $w$ model $\left(w(a)=w_{0}+w_{1}(1-a)\right)$. We constrain the models with the current observational data, including the type Ia supernova, the baryon acoustic oscillation, the cosmic microwave background, the Hubble expansion rate, and the X-ray gas mass fraction data. The fitting results indicate that a time-varying vacuum scenario is favored, in which the coupling $b(z)$ crosses the noninteracting line $(b=0)$ during the cosmological evolution and the sign changes from negative to positive. The crossing of the noninteracting line happens at around $z=0.2-0.3$, and the crossing behavior is favored at about $1 \sigma$ confidence level. Our work implies that we should pay more attention to the time-varying vacuum model and seriously consider the phenomenological construction of a sign-changeable or oscillatory interaction between dark sectors.
\end{abstract}

PACS numbers: 95.36.+x, 98.80.Es, 98.80.-k

\section{INTRODUCTION}

Dark energy (DE) and dark matter (DM) are the dominant components in the current universe, according to the recent astronomical observations [1-3]. They together account for about $96 \%$ of the critical energy density of the universe. However, ironically, we have known little about the natures of DE and DM. Although DM is "dark" (nonluminous), its gravitational property is normal, i.e., it gravitationally behaves like the usual baryon matter and thus can form structures in the universe. The property of DE is much more exotic in that it is gravitationally repulsive and so responsible for the accelerated expansion of the universe [4].

Since we are ignorant of the natures of both DE and DM, we cannot ignore such an important possibility that there is some direct, non-gravitational interaction between DE and DM. Intriguingly, such a possible interaction between DE and DM plays a crucial role in helping solve (or, at least alleviate) several important theoretical problems of DE. For example, it can be used to understand the cosmic coincidence problem [5], to avoid the cosmic doomsday brought by phantom [6], and to solve the cosmic age problem caused by old quasar [7] as well. Therefore, it is very meaningful to seriously study the interaction between dark sectors. Owing to the lack of the knowledge of micro-origin of the interaction, one has to first phenomenologically propose an interacting DE

\footnotetext{
*Electronic address: zhangxin@mail.neu.edu.cn
}

model and then test its theoretical and observational consequences. So far, lots of phenomenological interacting DE models have been studied $[8,9]$.

When considering the interaction between dark sectors, the continuity equations for energy densities of $\mathrm{DE}$ and DM are of the form:

$$
\begin{gathered}
\dot{\rho}_{d e}+3 H\left(1+w_{d e}\right) \rho_{d e}=-Q, \\
\dot{\rho}_{d m}+3 H \rho_{d m}=Q
\end{gathered}
$$

where $\rho_{d e}$ and $\rho_{d m}$ are the energy densities of DE and DM, respectively, $H=\dot{a} / a$ is the Hubble parameter, $a$ is the scale factor of the Friedmann-Robertson-Walker (FRW) universe, $w_{d e}=p_{d e} / \rho_{d e}$ is the equation of state (EOS) parameter of $\mathrm{DE}$, a dot denotes the derivative with respect to cosmic time $t$, and $Q$ denotes the phenomenological interaction term. Several forms for $Q$ have been put forward and have been fitted with observations [10]. Of course, all of these models are phenomenological. Most of them are constructed specifically for mathematical simplicity - for example, models in which $Q \propto H \rho$, where $\rho$ denotes the energy density of the dark sectors, and usually it has three choices, namely, $\rho=\rho_{d m}, \rho=\rho_{d e}$, and $\rho=\rho_{d e}+\rho_{d m}$. In addition, there are also some models [11] in which the assumption of that $Q$ is proportional to the Hubble parameter is abandoned and thus $Q \propto \rho$. Such models are designed by consulting the simple models of reheating, of dark matter decay into radiation, and of curvaton decay - i.e., where the interaction has the form of a decay of one species into another, with constant decay rate. However, it should 
be stressed that these models are severely dependent on the man-made choice of the special interaction forms. In other words, the predictions and observational consequences are model-dependent. In particular, the abovementioned phenomenological models exclude the important possibility that the interaction changes sign during the cosmological evolution. It is of interest to point out that a sign-changeable or oscillatory form of interaction is possible, according to the current observations.

Recently, Cai and Su [12], without choosing a special phenomenological form of interaction, proposed a novel scheme in which the whole redshift range is divided into a few bins and the interaction term $\delta(z)$ (note that here $Q=3 H \delta$ ) is set to be a constant in each bin, and by fitting the observational data they found that $\delta(z)$ is likely to cross the noninteracting $(\delta=0)$ line. This study is fairly enlightening and inspires us to open our mind to seriously consider the possibility of that the interaction between dark sectors changes sign during the cosmological evolution. If such an observation is conclusive, it is suggested that more general phenomenological forms of interaction term should be put forth. However, the work of Cai and $\mathrm{Su}$ [12] seems not sufficient to prove that the interaction changes its sign, since their conclusion is drawn based upon the behavior of the best-fitted $\delta(z)$, and the errors of the fitting results weaken the conclusion to a great degree due to the fact that the observational data currently available cannot determine more than two parameters in a piecewise parametrization approach.

In this paper, our aim is to verify whether the interaction indeed changes its sign (i.e., crosses the noninteracting line) during the evolution, by using a different method. We propose a parametrization form for the interaction term $Q$. In our work, we further abandon the assumption that $Q$ is proportional to the Hubble expansion rate $H$. So, the interaction term $Q$ is only characterized by the coupling $b$ :

$$
Q(a)=3 b(a) H_{0} \rho_{0},
$$

where the dimensionless coupling $b(a)$ is variable with the cosmological evolution. Note that here the occurrence of the present-day Hubble parameter $H_{0}$ and the present-day density of dark sectors $\rho_{0}=\rho_{d e 0}+\rho_{d m 0}$ is only for a dimensional reason. By the way, in the whole work the subscript " 0 " always indicates the present-day value of the corresponding quantity. From the form of Eq. (3), we see that the evolution of the interaction term $Q$ is totally described by the running of the coupling constant, $b(a)$, so our scenario can be called the "running coupling". Furthermore, we assume that the coupling $b$ is described by a constant $b_{0}$ at the late times, and determined by another constant $b_{e}$ at the early times; and the whole evolution of $b(a)$ is totally characterized by the two parameters, $b_{0}$ and $b_{e}$. For continuously connecting the early-time and late-time behaviors, we put forward the following two-parameter form for the coupling $b(a)$ :

$$
b(a)=b_{0} a+b_{e}(1-a) .
$$

Though the interaction term in our work depends on a particular parametrization form, the parameters can be tightly constrained by the current observational data, overcoming the disadvantage of the piecewise fitting method. The reconstructed evolution of $b(a)$ will indicate whether the coupling between the dark sectors crosses the noninteracting line.

In this paper, we will investigate our coupling parametrization with the latest observational data. For the EOS of DE, $w$, we consider the following three cases: (1) the cosmological constant (vacuum energy), $w=-1$; (2) the constant EOS, $w=w_{0}$; (3) the timevariable EOS, namely, the Chevallier-Polarski-Linder $(\mathrm{CPL})$ parametrization, $w(a)=w_{0}+w_{1}(1-a)$ [13]. We will fit the three interacting DE models with the data from the Union2 type Ia supernovae (SNIa), the baryon acoustic oscillation (BAO), the cosmic microwave background (CMB), the Hubble expansion rate, and the Xray gas mass fraction. We obtain the best-fitted parameters and likelihoods by using the Monte Carlo Markov chain (MCMC) method. We will show that the interaction $Q$ between $\mathrm{DE}$ and $\mathrm{DM}$ indeed changes sign around $z=0.2-0.3$ during the cosmological evolution, at about $1 \sigma$ confidence level (CL).

\section{METHODOLOGY}

We consider interacting DE models in a spatially flat FRW universe. The Friedmann equation reads

$$
3 M_{P l}^{2} H^{2}=\rho_{r}+\rho_{b}+\rho_{d e}+\rho_{d m},
$$

where $\rho_{r}, \rho_{b}, \rho_{d e}$ and $\rho_{d m}$ are the energy densities of radiation, baryon, $\mathrm{DE}$ and $\mathrm{DM}$, respectively, and $M_{P l}$ is the reduced Planck mass. It is convenient to introduce the fractional energy densities $\Omega_{i} \equiv \rho_{i} / 3 M_{P l}^{2} H^{2}$, with $i=r, b, d e$, and $d m$. Obviously,

$$
\Omega_{r}+\Omega_{b}+\Omega_{d e}+\Omega_{d m}=1 .
$$

Substituting Eqs. (3) and (4) into Eqs. (1) and (2), and defining the functions $f_{d e}=\rho_{d e} / \rho_{d e 0}$ and $f_{d m}=$ $\rho_{d m} / \rho_{d m 0}$, we obtain

$$
\frac{d f_{d e}(x)}{d x}+3(1+w) f_{d e}(x)=-\frac{3}{E(x)}\left(1+\frac{1}{f_{0}}\right)\left[b_{0} e^{x}+b_{e}\left(1-e^{x}\right)\right],
$$

$\frac{d f_{d m}(x)}{d x}+3 f_{d m}(x)=\frac{3}{E(x)}\left(1+f_{0}\right)\left[b_{0} e^{x}+b_{e}\left(1-e^{x}\right)\right]$,

where $x \equiv \ln a, f_{0} \equiv \rho_{d e 0} / \rho_{d m 0}=\Omega_{d e 0} / \Omega_{d m 0}=$ $\left(1-\Omega_{r 0}-\Omega_{b 0}-\Omega_{d m 0}\right) / \Omega_{d m 0}$, and $E(x) \equiv H(x) / H_{0}=$ $\left[\Omega_{r 0} e^{-4 x}+\Omega_{b 0} e^{-3 x}+\Omega_{d m 0} f_{d m}(x)+\left(1-\Omega_{r 0}-\Omega_{b 0}-\right.\right.$ $\left.\left.\Omega_{d m 0}\right) f_{d e}(x)\right]^{1 / 2}$. Therefore, given the values of the parameters $\Omega_{r 0}, \Omega_{b 0}, \Omega_{d m 0}$ and $w$, Eqs. (7) and (8) can be numerically solved with the initial conditions $f_{d e}(0)=1$ and $f_{d m}(0)=1$. With the resulting functions $f_{d e}(x)$ 
and $f_{d m}(x)$, we finally obtain the function $E(x)$, the dimensionless Hubble expansion rate. As aforementioned, in this work we employ three DE models, namely, the cosmological constant model $(\Lambda \mathrm{CDM})$ with $w=-1$, the constant EOS model (XCDM) with $w=w_{0}$, and the time-variable EOS model (CPL) with $w(x)=w_{0}+$ $w_{1}\left(1-e^{x}\right)$.

To fit the three interacting DE models with observations, we use the data from the Union2 SNIa (557 data), the BAO from SDSS DR7, the CMB from 7-year WMAP, the Hubble expansion rate (15 data), and the $\mathrm{X}$-ray gas mass fraction (42 data). The best-fitted parameters are obtained by minimizing the sum

$$
\chi^{2}=\tilde{\chi}_{S N}^{2}+\chi_{B A O}^{2}+\chi_{C M B}^{2}+\chi_{H}^{2}+\chi_{X-r a y}^{2} .
$$

We obtain the constraints by using a MCMC method.

Supernovae.- We use the data points of the 557 Union2 SNIa compiled in Ref. [14]. The theoretical distance modulus is defined as

$$
\mu_{t h}\left(z_{i}\right) \equiv 5 \log _{10} D_{L}\left(z_{i}\right)+\mu_{0}
$$

where $z=1 / a-1$ is the redshift, $\mu_{0} \equiv 42.38-5 \log _{10} h$ with $h$ the Hubble constant $H_{0}$ in units of $100 \mathrm{~km} / \mathrm{s} / \mathrm{Mpc}$, and the Hubble-free luminosity distance

$$
D_{L}(z)=(1+z) \int_{0}^{z} \frac{d z^{\prime}}{E\left(z^{\prime} ; \boldsymbol{\theta}\right)}
$$

where $\boldsymbol{\theta}$ denotes the model parameters. Correspondingly, the $\chi^{2}$ function for the 557 Union2 SNIa data is given by

$$
\chi_{S N}^{2}(\boldsymbol{\theta})=\sum_{i=1}^{557} \frac{\left[\mu_{o b s}\left(z_{i}\right)-\mu_{t h}\left(z_{i}\right)\right]^{2}}{\sigma^{2}\left(z_{i}\right)},
$$

where $\sigma$ is the corresponding $1 \sigma$ error of distance modulus for each supernova. The parameter $\mu_{0}$ is a nuisance parameter and one can expand Eq. (12) as

$$
\chi_{S N}^{2}(\boldsymbol{\theta})=A(\boldsymbol{\theta})-2 \mu_{0} B(\boldsymbol{\theta})+\mu_{0}^{2} C,
$$

where $A(\boldsymbol{\theta}), B(\boldsymbol{\theta})$ and $C$ are defined in Ref. [15]. Evidently, Eq. (13) has a minimum for $\mu_{0}=B / C$ at

$$
\tilde{\chi}_{S N}^{2}(\boldsymbol{\theta})=A(\boldsymbol{\theta})-\frac{B(\boldsymbol{\theta})^{2}}{C} .
$$

Since $\chi_{S N, \min }^{2}=\tilde{\chi}_{S N, \min }^{2}$, instead minimizing $\chi_{S N}^{2}$ we will minimize $\tilde{\chi}_{S N}^{2}$ which is independent of the nuisance parameter $\mu_{0}$.

Baryon acoustic oscillations.- We use the BAO data from SDSS DR7 [16]. The distance ratio $\left(d_{z}\right)$ at $z=0.2$ and $z=0.35$ are

$$
d_{0.2}=\frac{r_{s}\left(z_{d}\right)}{D_{V}(0.2)}, \quad d_{0.35}=\frac{r_{s}\left(z_{d}\right)}{D_{V}(0.35)},
$$

where $r_{s}\left(z_{d}\right)$ is the comoving sound horizon at the baryon drag epoch [17], and

$$
D_{V}(z)=\left[\left(\int_{0}^{z} \frac{d z^{\prime}}{H\left(z^{\prime}\right)}\right)^{2} \frac{z}{H(z)}\right]^{1 / 3}
$$

encodes the visual distortion of a spherical object due to the non Euclidianity of a FRW spacetime. The inverse covariance matrix of $\mathrm{BAO}$ is

$$
\left(C_{B A O}^{-1}\right)=\left(\begin{array}{cc}
30124 & -17227 \\
-17227 & 86977
\end{array}\right)
$$

The $\chi^{2}$ function of the BAO data is constructed as:

$$
\chi_{B A O}^{2}=\left(d_{i}^{t h}-d_{i}^{o b s}\right)\left(C_{B A O}^{-1}\right)_{i j}\left(d_{j}^{t h}-d_{j}^{o b s}\right),
$$

where $d_{i}=\left(d_{0.2}, d_{0.35}\right)$ is a vector, and the BAO data we use are $d_{0.2}=0.1905$ and $d_{0.35}=0.1097$.

Cosmic microwave background.- We employ the "WMAP distance priors" given by the 7-year WMAP observations [18]. This includes the "acoustic scale" $l_{A}$, the "shift parameter" $R$, and the redshift of the decoupling epoch of photons $z_{*}$. The acoustic scale $l_{A}$ describes the distance ratio $D_{A}\left(z_{*}\right) / r_{s}\left(z_{*}\right)$, defined as

$$
l_{A} \equiv\left(1+z_{*}\right) \frac{\pi D_{A}\left(z_{*}\right)}{r_{s}\left(z_{*}\right)}
$$

where a factor of $\left(1+z_{*}\right)$ arises because $D_{A}\left(z_{*}\right)$ is the proper angular diameter distance, whereas $r_{s}\left(z_{*}\right)$ is the comoving sound horizon at $z_{*}$. The fitting formula of $r_{s}(z)$ is given by

$$
r_{s}(z)=\frac{1}{\sqrt{3}} \int_{0}^{1 /(1+z)} \frac{d a}{a^{2} H(a) \sqrt{1+\left(3 \Omega_{b 0} / 4 \Omega_{\gamma 0}\right) a}} .
$$

In this paper, we fix $\Omega_{\gamma 0}=2.469 \times 10^{-5} h^{-2}, \Omega_{b 0}=$ $0.02246 h^{-2}$, given by the 7-year WMAP observations [18], and $\Omega_{r 0}=\Omega_{\gamma 0}\left(1+0.2271 N_{\text {eff }}\right)$ with $N_{\text {eff }}$ the effective number of neutrino species (in this paper we take its standard value, 3.04 [18]). We use the fitting function of $z_{*}$ proposed by $\mathrm{Hu}$ and Sugiyama [19]

$$
z_{*}=1048\left[1+0.00124\left(\Omega_{b 0} h^{2}\right)^{-0.738}\right]\left[1+g_{1}\left(\Omega_{m 0} h^{2}\right)^{g_{2}}\right],
$$

where $\Omega_{m 0}=\Omega_{b 0}+\Omega_{d m 0}$ and

$$
g_{1}=\frac{0.0783\left(\Omega_{b 0} h^{2}\right)^{-0.238}}{1+39.5\left(\Omega_{b 0} h^{2}\right)^{0.763}}, \quad g_{2}=\frac{0.560}{1+21.1\left(\Omega_{b 0} h^{2}\right)^{1.81}} .
$$

The shift parameter $R$ is responsible for the distance ratio $D_{A}\left(z_{*}\right) / H^{-1}\left(z_{*}\right)$, given by $[20]$

$$
R\left(z_{*}\right) \equiv \sqrt{\Omega_{m 0} H_{0}^{2}}\left(1+z_{*}\right) D_{A}\left(z_{*}\right) .
$$

Following [18], we use the prescription for using the WMAP distance priors. Thus, the $\chi^{2}$ function for the CMB data is

$$
\chi_{C M B}^{2}=\left(x_{i}^{t h}-x_{i}^{o b s}\right)\left(C_{C M B}^{-1}\right)_{i j}\left(x_{j}^{t h}-x_{j}^{o b s}\right),
$$

where $x_{i}=\left(l_{A}, R, z_{*}\right)$ is a vector, and $\left(C_{C M B}^{-1}\right)_{i j}$ is the inverse covariance matrix. The 7-year WMAP observations [18] give the maximum likelihood values: $l_{A}\left(z_{*}\right)=$ 
302.09, $R\left(z_{*}\right)=1.725$, and $z_{*}=1091.3$. The inverse covariance matrix is also given in [18]:

$$
\left(C_{C M B}^{-1}\right)=\left(\begin{array}{ccc}
2.305 & 29.698 & -1.333 \\
29.698 & 6825.27 & -113.180 \\
-1.333 & -113.180 & 3.414
\end{array}\right)
$$

Hubble expansion rate.- For the Hubble parameter $H(z)$, there are 15 observational data available, where twelve of them are from Ref. [21]. In addition, in Ref. [22], the authors obtain the additional three data: $H(z=0.24)=79.69 \pm 2.32, H(z=0.34)=83.8 \pm 2.96$, and $H(z=0.43)=86.45 \pm 3.27$ (in units of $\mathrm{km} \mathrm{s}^{-1}$ $\left.\mathrm{Mpc}^{-1}\right)$. The $\chi^{2}$ function for the observational Hubble data is

$$
\chi_{H}^{2}(\boldsymbol{\theta})=\sum_{i=1}^{15} \frac{\left[H_{t h}\left(\boldsymbol{\theta} ; z_{i}\right)-H_{o b s}\left(z_{i}\right)\right]^{2}}{\sigma^{2}\left(z_{i}\right)} .
$$

$X$-ray gas mass fraction.- For the $f_{\text {gas }}$ data, we use the Chandra measurements in Ref. [23]. In the framework of the $\Lambda$ CDM reference cosmology, the X-ray gas mass fraction is presented as $[23,24]$

$$
f_{\text {gas }}(z)=\frac{K A \gamma b(z)}{1+s(z)}\left(\frac{\Omega_{b 0}}{\Omega_{b 0}+\Omega_{d m 0} F(z)}\right)\left[\frac{D_{A}^{\Lambda C D M}(z)}{D_{A}(z)}\right]^{1.5}
$$

where the effect of the interaction between dark sectors has been considered, resulting in an additional function $F(z)=f_{d m}(-\ln (1+z)) /(1+z)^{3}$. The parameters $K, \gamma$, $b(z)$ and $s(z)$ model the abundance of gas in the clusters. We set these parameters to their respective best-fit values of Ref. [23]. $A$ is the angular correction factor, which is caused by the change in angle for the current test model $\theta_{2500}$ in comparison with that of the reference cosmology $\theta_{2500}^{\Lambda C D M}$ :

$$
A=\left(\frac{\theta_{2500}^{\Lambda C D M}}{\theta_{2500}}\right)^{\eta} \approx\left(\frac{H(z) D_{A}(z)}{\left[H(z) D_{A}(z)\right]^{\Lambda C D M}}\right)^{\eta}
$$

here, the index $\eta$ is the slope of the $f_{g a s}\left(r / r_{2500}\right)$ data within the radius $r_{2500}$, with the best-fit average value $\eta=0.214 \pm 0.022[23]$. And the proper (not comoving) angular diameter distance is given by

$$
D_{A}(z)=\frac{1}{(1+z)} \int_{0}^{z} \frac{d z^{\prime}}{H\left(z^{\prime}\right)}
$$

The $\chi^{2}$ function for the $f_{\text {gas }}$ data from the 42 galaxy clusters reads

$$
\chi_{X-r a y}^{2}(\boldsymbol{\theta})=\sum_{i=1}^{42} \frac{\left(\left[f_{\text {gas }}\left(\boldsymbol{\theta} ; z_{i}\right)\right]_{t h}-\left[f_{\text {gas }}\left(z_{i}\right)\right]_{\text {obs }}\right)^{2}}{\sigma^{2}\left(z_{i}\right)} .
$$

It should be pointed out that the $f_{\text {gas }}$ data are rather crucial for the fitting, since they can be used to break the degeneracy between the parameters from the interaction and the EOS of CPL model. So, the inclusion of the $f_{\text {gas }}$ data in our fitting is indispensable.

\section{RESULTS}

Now we fit our interacting models with the observations. For the interacting $\Lambda \mathrm{CDM}, \mathrm{XCDM}$ and $\mathrm{CPL}$ models, the parameters are $\boldsymbol{\theta}=\left\{\Omega_{d m 0}, b_{0}, b_{e}, h\right\}$, $\left\{\Omega_{d m 0}, w_{0}, b_{0}, b_{e}, h\right\}$ and $\left\{\Omega_{d m 0}, w_{0}, w_{1}, b_{0}, b_{e}, h\right\}$, respectively. We use the MCMC method and finally we obtain the best-fit parameters and the corresponding $\chi_{\text {min }}^{2}$. The best-fit, $1 \sigma$ and $2 \sigma$ values of the parameters with $\chi_{\text {min }}^{2}$ of the three interacting models are all presented in Table I.
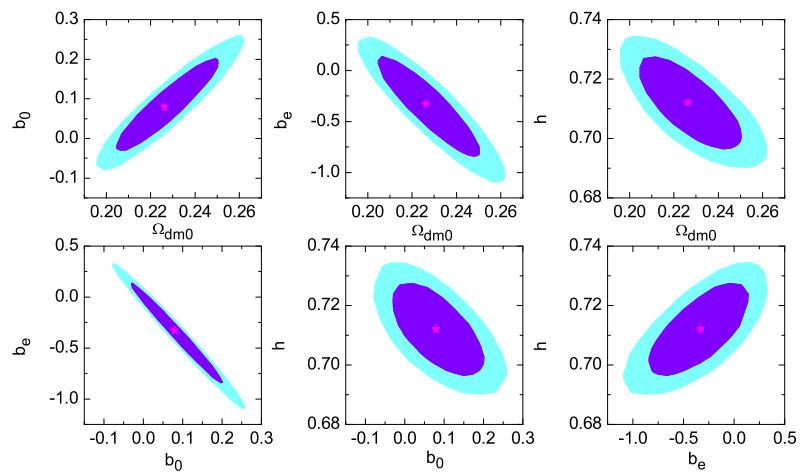

FIG. 1: The probability contours at $1 \sigma$ and $2 \sigma$ confidence levels in the parameter planes for the interacting $\Lambda \mathrm{CDM}$ model.

Figure 1 shows the likelihood contours for the interacting $\Lambda$ CDM model. For this model, we have $\Omega_{d m 0}=$ $0.2262, b_{0}=0.0793, b_{e}=-0.3274$ and $h=0.7120$, with $\chi_{\min }^{2}=595.968$. We plot the likelihood contours for the interacting XCDM model in Fig. 2. For the interacting XCDM model, the fitting results are $\Omega_{d m 0}=$ $0.2267, w_{0}=-0.9844, b_{0}=0.0787, b_{e}=-0.3216$ and $h=0.7094$, with $\chi_{\min }^{2}=595.815$. For the interacting CPL model, we obtain the fitting results: $\Omega_{d m 0}=0.2271$, $w_{0}=-0.9768, w_{1}=-0.0455 b_{0}=0.0799, b_{e}=-0.3290$ and $h=0.7097$, with $\chi_{\min }^{2}=595.808$. The likelihood contours for this case are shown in Fig. 3.

From the fitting results, we first notice that the EOS of $\mathrm{DE}$ is near -1 in both the constant $w$ and time-dependent $w$ scenarios. In the constant $w$ case, we get the best-fitted value $w_{0}=-0.9844$, rather close to -1 , albeit slightly tends to a quintessence $(w>-1)$. For the CPL case, the best-fitted values are $w_{0}=-0.9768$ and $w_{1}=-0.0455$; we also notice that $w_{0}$ is fairly near -1 and $w_{1}$ is close to 0 . So, in the interacting DE model with a running coupling, even if the EOS of DE is allowed to vary, the data still favor a slightly evolving EOS with the value approaching -1 . To see this clearly, we plot the reconstructed $w(z)$ for the interacting CPL model in Fig. 4 where the best fit and the $1 \sigma$ uncertainties are shown. According the fitting results, we realize that a timevarying vacuum scenario is favored by the data. The time-varying vacuum model has been discussed extensively $[25,26]$. Our work indicates that the scenario of a time-varying vacuum with a running coupling deserves 
TABLE I: The fitting results of the parameters with best-fit values as well as $1 \sigma$ and $2 \sigma$ errors in the three interacting DE models.

\begin{tabular}{|c|c|c|c|}
\hline model parameters & $\overline{\Lambda \mathrm{CDM}}$ & $\overline{\mathrm{XCDM}}$ & $\overline{\mathrm{CPL}}$ \\
\hline$\Omega_{d m 0}$ & $0.2262_{-0.0215-0.0304}^{+0.0242+0.0356}$ & $0.2267_{-0.0237-0.0327}^{+0.0273+0.0388}$ & $0.2271_{-0.0280-0.0360}^{+0.0302+0.0443}$ \\
\hline$w_{0}$ & $\mathrm{~N} / \mathrm{A}$ & $-0.9844_{-0.0932-0.1357}^{+0.0915+0.1282}$ & $-0.9768_{-0.2090-0.2635}^{+0.3046+0.4322}$ \\
\hline$w_{1}$ & $\mathrm{~N} / \mathrm{A}$ & $\mathrm{N} / \mathrm{A}$ & $-0.0455_{-1.8125-2.7810}^{+0.9426+1.2742}$ \\
\hline$b_{0}$ & $0.0793_{-0.1092-0.1580}^{+0.1220+0.1797}$ & $0.0787_{-0.1159-0.1654}^{+0.1353+0.1962}$ & $0.0799_{-0.1342-0.1894}^{+0.152+0.2039}$ \\
\hline$b_{e}$ & $-0.3274_{-0.5140-0.7644}^{+0.4646+0.6543}$ & $-0.3216_{-0.5771-0.8317}^{+0.5007+0.7082}$ & $-0.3290_{-0.6447-0.8989}^{+0.5855+0.8379}$ \\
\hline$h$ & $0.7120_{-0.0155-0.0218}^{+0.0154+0.0224}$ & $0.7094_{-0.0219-0.0315}^{+0.0224+0.0324}$ & $0.7097_{-0.0257-0.0357}^{+0.0259+0.0348}$ \\
\hline$\chi_{\min }^{2}$ & 595.968 & 595.815 & 595.808 \\
\hline
\end{tabular}

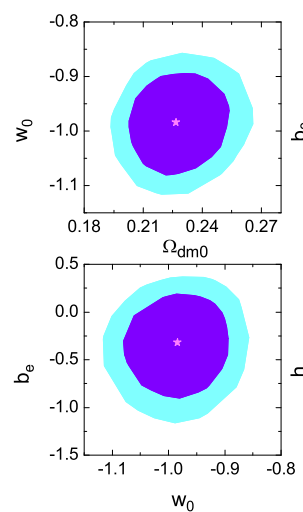

$\mathrm{w}_{0}$
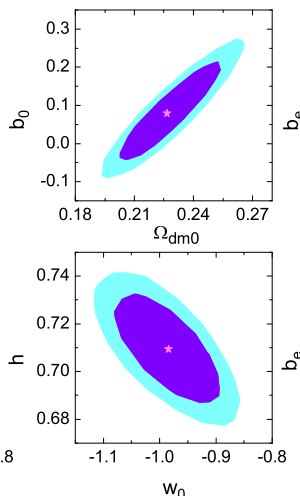

$\mathrm{w}_{0}$
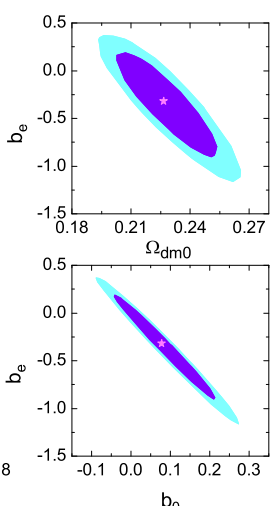
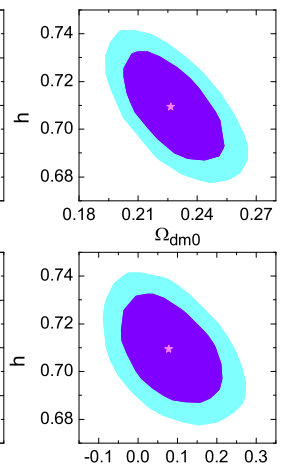

$b_{0}$
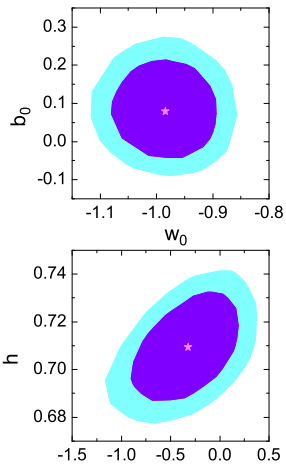

$b_{e}$

FIG. 2: The probability contours at $1 \sigma$ and $2 \sigma$ confidence levels in the parameter planes for the interacting XCDM model.

more further investigations.

For the running coupling $b(z)$, we obtain similar results in all the three scenarios. From Figs. 1-3 we see that the parameters $b_{0}$ and $b_{e}$ are in strong anti-correlation. The best-fitted values for $b_{0}$ and $b_{e}$ are: $b_{0} \approx 0.08$ and $b_{e} \approx-0.3$, implying that the coupling $b(z)$ crosses the noninteracting line $b=0$ during the cosmological evolution, and the sign changes from negative to positive. Such a feature for the interaction is favored by the data at about $1 \sigma$ level. To see the crossing phenomenon clearly, we reconstruct the evolution of the coupling $b(z)$ by using a Fisher Matrix technique, shown in Fig. 5. From this figure we read out that the crossing happens at $z=0.2-0.3$. Our results tell such a story: at early times when DM dominates the universe, the energy transfer direction is from $\mathrm{DM}$ to $\mathrm{DE}$, and at late times when $\mathrm{DE}$ becomes dominant, the decay direction reverses, from DE to DM. The above phenomenon is favored at $1 \sigma$ CL. Note that once the reconstruction of $b(z)$ is performed based on a Monte Carlo method, the fluctuations will become larger, but the above distinctive feature still stands by at about $1 \sigma$ CL.

In the work of Cai and $\mathrm{Su}$ [12], a redshift-binned parametrization method is used, but the limitation is that it is hard to go beyond two parameters for tight constraints. Also, in Ref. [12], as the data give only weak constraint for $z>1.8$, the additional assumption $Q=0$ for $1.8<z<1090$ is made. In our method, the parametrization $Q(a)=3 b(a) H_{0} \rho_{0}$ with $b(a)=b_{0} a+b_{e}(1-a)$ has only two parameters, and the early-time interaction can also be described. Our fitting results support the conclusion of Cai and $\mathrm{Su}$ [12] that the interaction $Q(z)$ crosses the noninteracting line, in a distinct way. The limitations of our parametrization are: (1) whether or not there is some oscillation feature in the interaction cannot be read out, and (2) the future evolution cannot be described owing to the fact that $b(z)$ will diverge as $z$ approaches -1 , so the predictive power of this parametrization is lost (see [27] for a similar case for $w(z))$. We will go beyond these limitations in the future work. In addition, now that the analysis of the current observational data provides a hint that the interaction between dark sectors might change sign during the cosmological evolution, more general phenomenological forms for the interaction describing the sign-changeable or oscillatory feature should be seriously considered.

\section{CONCLUSION}

Since the knowledge about the micro-origin of dark sector interaction is absent, one has no way to know the form of the interaction term $Q$ from a microscopic theory. A popular way to investigate the interacting dark energy is to assume a specific phenomenological form for $Q$; for instance, $Q \propto H \rho$ or $Q \propto \rho$, where $\rho$ denotes the energy 

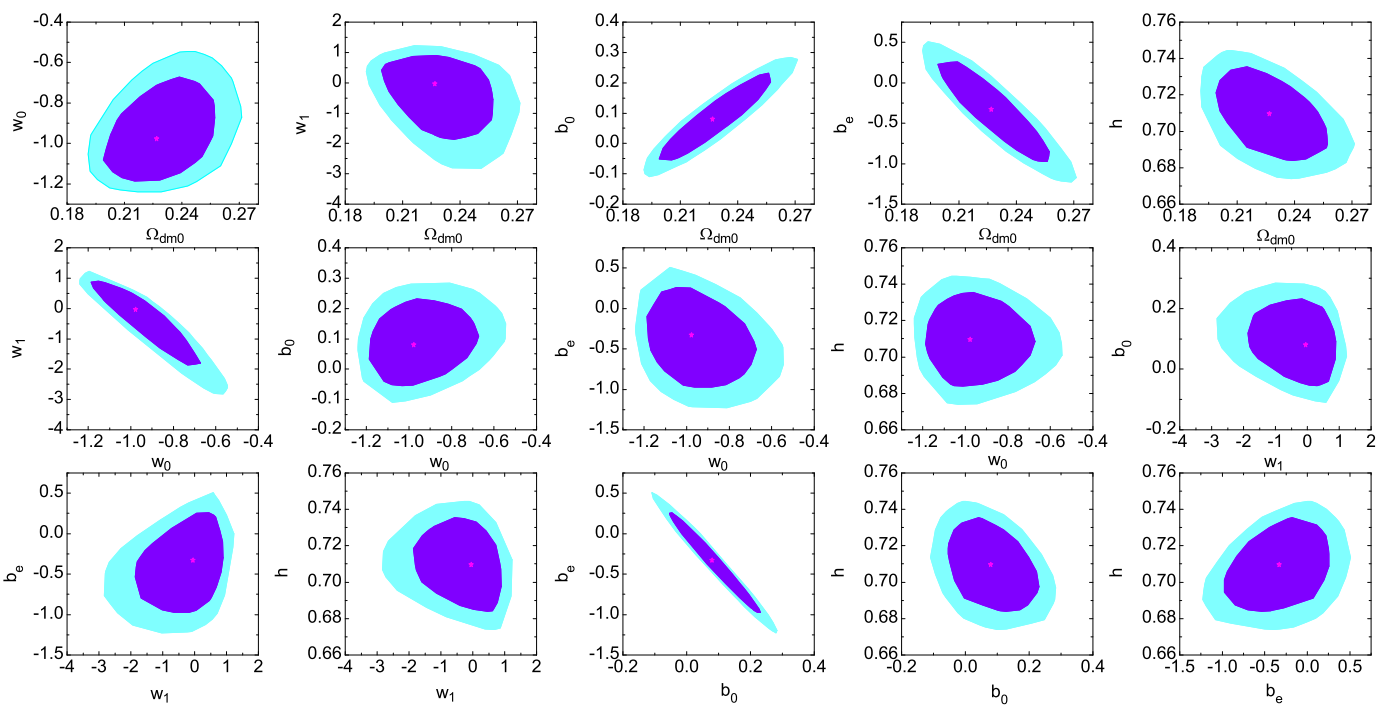

FIG. 3: The probability contours at $1 \sigma$ and $2 \sigma$ confidence levels in the parameter planes for the interacting CPL model.

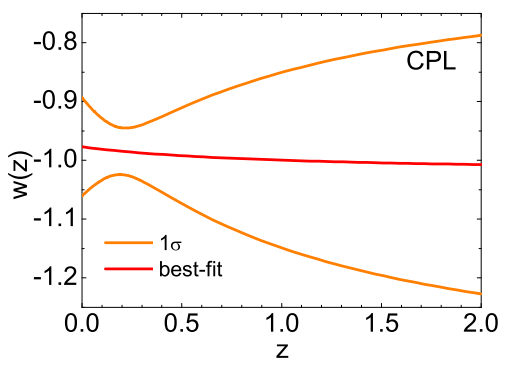

FIG. 4: The reconstructed evolutionary history for $w(z)$ of the interacting CPL model.

density of dark sectors. However, the choice of the phenomenological forms is rather arbitrary. In the face of this situation, let us recall the method used to probe the dynamical evolution of the EOS of DE, $w(z)$, with the observational data. Owing to our ignorance of $\mathrm{DE}$, for probing the dynamical evolution of $w$, one has to parameterize $w$ empirically, usually using two parameters, e.g., the most widely used CPL form, $w(a)=w_{0}+w_{1}(1-a)$. Inspired by this method, we try to parameterize the evolution of $Q$ in a similar way, and then use the observational data to probe the dynamical evolution of $Q$. Such a way may provide a guidance for finding a reasonable phenomenological form for $Q$.

In this paper, we have put forward a running coupling scenario for describing the interaction between $\mathrm{DE}$ and DM. In this scenario, the dark sector interaction has the form $Q(a)=3 b(a) H_{0} \rho_{0}$, where $b(a)$ is the coupling which is variable during the cosmological evolution. We have proposed a parametrization form for the running coupling: $b(a)=b_{0} a+b_{e}(1-a)$. So, at the early times the coupling is given by a constant $b_{e}$, while today the coupling is described by another constant, $b_{0}$. We have constrained the parameters $b_{0}$ and $b_{e}$ with the observational data currently available, including the Union2 SNIa, BAO (from SDSS DR7), CMB (from 7year WMAP), $H(z)$, and X-ray gas mass fraction data. It should be stressed that the $f_{\text {gas }}$ data are very crucial in our analysis since they are helpful in breaking the degeneracy between the parameters. And, in our analysis, we employed three DE model, namely, the $\Lambda \mathrm{CDM}$, XCDM and CPL models.

Our fitting results show that the EOS of DE, $w$, is close to -1 both in the constant $w$ (XCDM) and timedependent $w(\mathrm{CPL})$ cases. Thus, a time-varying vacuum scenario is favored by the data, according to this analysis. In addition, for the running coupling $b(z)$, the results are also similar in all the three DE models. The parameters $b_{0}$ and $b_{e}$ are in strong anti-correlation, and the best-fitted values are: $b_{0} \approx 0.08$ and $b_{e} \approx-0.3$. This implies that the coupling $b(z)$ crosses the noninteracting line $(b=0)$ and the sign changes from $b<0$ to $b>0$. The reconstruction of $b(z)$ indicates that the crossing of the noninteracting line happens at around $z=0.2-0.3$, and the crossing behavior is favored at about $1 \sigma$ CL. Therefore, our work tells a story about the interacting DE model: $\mathrm{DE}$ is a time-varying vacuum; the coupling between $\mathrm{DE}$ and DM runs with the expansion of the universe; at early times when DM dominates the universe, DM decays to $\mathrm{DE}$, while at late times when $\mathrm{DE}$ becomes dominant, $\mathrm{DE}$ begins to decay to DM. If the above scenario is true, then we should pay more attention to the time-varying vacuum model, and seriously consider how to construct a sign-changeable interaction between dark sectors phenomenologically. For the time-varying vacuum model, previous work neglects the perturbations of DE and only treats the decaying vacuum as a background, however, if DE interacts with DM, the perturbations of DE should also be taken into account even if $w=-1$. This deserves further investigations.

Finally, we discuss the limitations of our parametriza- 

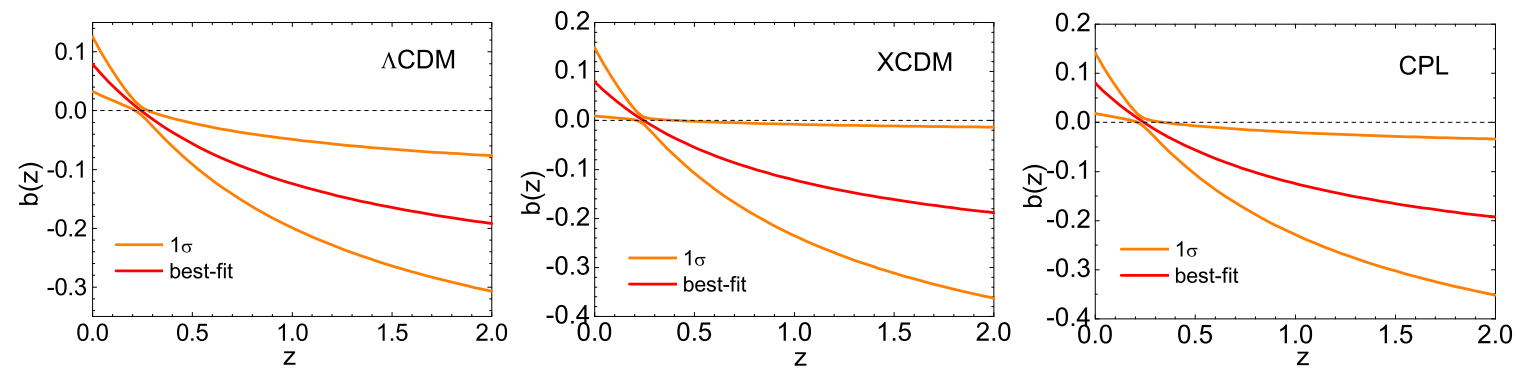

FIG. 5: The reconstructed evolutionary histories for $b(z)$ in the three interacting models. The dashed line in each plot represents the noninteracting line.

tion for the running coupling. Our parametrization cannot describe the possible oscillation in the dark sector interaction. Moreover, such a parametrization form cannot predict the future evolution of the interaction, since the coupling $b(z)$ will encounter divergency in the far future. We will go beyond these limitations in our future work.

\section{Acknowledgments}

This work was supported by the Natural Science Foundation of China under Grant Nos. 10705041 and 10975032, as well as the National Innovation Experiment Program for University Students.
[1] A. G. Riess et al. [Supernova Search Team Collaboration], Astron. J. 116, 1009 (1998) [astro-ph/9805201]; S. Perlmutter et al. [Supernova Cosmology Project Collaboration], Astrophys. J. 517, 565 (1999) [astro$\mathrm{ph} / 9812133]$.

[2] M. Tegmark et al. [SDSS Collaboration], Phys. Rev. D 69, 103501 (2004) [astro-ph/0310723]; K. Abazajian et al. [SDSS Collaboration], Astron. J. 128, 502 (2004) [astro-ph/0403325]; K. Abazajian et al. [SDSS Collaboration], Astron. J. 129, 1755 (2005) [astro-ph/0410239].

[3] D. N. Spergel et al. [WMAP Collaboration], Astrophys. J. Suppl. 148175 (2003) [astro-ph/0302209].

[4] V. Sahni and A. A. Starobinsky, Int. J. Mod. Phys. D 9, 373 (2000) [arXiv:astro-ph/9904398]; P. J. E. Peebles and B. Ratra, Rev. Mod. Phys. 75, 559 (2003) [arXiv:astroph/0207347]; V. Sahni, Lect. Notes Phys. 653, 141 (2004) [arXiv:astro-ph/0403324]; E. J. Copeland, M. Sami and S. Tsujikawa, Int. J. Mod. Phys. D 15, 1753 (2006) [arXiv:hep-th/0603057]; J. Frieman, M. Turner and D. Huterer, Ann. Rev. Astron. Astrophys. 46, 385 (2008) [arXiv:0803.0982 [astro-ph]]; S. Nojiri and S. D. Odintsov, Phys. Rept. (2011) doi: 10.1016/j.physrep.2011.04.001 [arXiv:1011.0544 [gr-qc]]; M. Li, X. D. Li, S. Wang and Y. Wang, arXiv:1103.5870 [astro-ph.CO].

[5] L. Amendola, Phys. Rev. D 62, 043511 (2000) [arXiv:astro-ph/9908023]; D. Comelli, M. Pietroni and A. Riotto, Phys. Lett. B 571, 115 (2003) [arXiv:hepph/0302080]; X. Zhang, Mod. Phys. Lett. A 20, 2575 (2005) [arXiv:astro-ph/0503072]; R. G. Cai and A. Wang, JCAP 0503, 002 (2005) [arXiv:hep-th/0411025].

[6] E. Elizalde, S. Nojiri and S. D. Odintsov, Phys. Rev. D 70, 043539 (2004) [arXiv:hep-th/0405034]; S. Nojiri, S. D. Odintsov and S. Tsujikawa, Phys. Rev. D 71, 063004 (2005) [arXiv:hep-th/0501025]; S. Nojiri and S. D. Odintsov, Phys. Lett. B 595, 1 (2004) [arXiv:hepth/0405078]; M. Li, C. Lin and Y. Wang, JCAP 0805,
023 (2008) [arXiv:0801.1407 [astro-ph]]; M. Li, X. D. Li, S. Wang, Y. Wang and X. Zhang, JCAP 0912, 014 (2009) [arXiv:0910.3855 [astro-ph.CO]].

[7] S. Wang and Y. Zhang, Phys. Lett. B 669, 201 (2008) [arXiv:0809.3627 [astro-ph]]; J. Cui and X. Zhang, Phys. Lett. B 690, 233 (2010) [arXiv:1005.3587 [astroph.CO]]; Y. H. Li, J. Z. Ma, J. L. Cui, Z. Wang and X. Zhang, Sci. China Phys. Mech. Astron. (2011) doi: 10.1007/s11433-011-4382-1 [arXiv:1011.6122 [astroph.CO]]; I. Duran and D. Pavon, Phys. Rev. D 83, 023504 (2011) [arXiv:1012.2986 [astro-ph.CO]].

[8] S. Capozziello, S. Nojiri and S. D. Odintsov, Phys. Lett. B 632, 597 (2006) [arXiv:hep-th/0507182]; S. Nojiri and S. D. Odintsov, Gen. Rel. Grav. 38, 1285 (2006) [arXiv:hep-th/0506212]; S. Nojiri and S. D. Odintsov, Phys. Rev. D 72, 023003 (2005) [arXiv:hep-th/0505215].

[9] B. Wang, Y. Gong and E. Abdalla, Phys. Lett. B 624, 141 (2005) [arXiv:hep-th/0506069]; B. Wang, C. Y. Lin, D. Pavon and E. Abdalla, Phys. Lett. B 662, 1 (2008) [arXiv:0711.2214 [hep-th]]; X. Zhang, Phys. Lett. B 611, 1 (2005) [arXiv:astro-ph/0503075]; X. Zhang, Commun. Theor. Phys. 44, 762 (2005); J. Zhang, H. Liu and X. Zhang, Phys. Lett. B 659, 26 (2008) [arXiv:0705.4145 [astro-ph]]; H. Wei and R. G. Cai, Eur. Phys. J. C 59, 99 (2009) [arXiv:0707.4052 [hep-th]]; L. Zhang, J. Cui, J. Zhang and X. Zhang, Int. J. Mod. Phys. D 19, 21 (2010) [arXiv:0911.2838 [astro-ph.CO]]; M. Suwa and T. Nihei, Phys. Rev. D 81, 023519 (2010) [arXiv:0911.4810 [astro-ph.CO]]; S. Chen, B. Wang and J. Jing, Phys. Rev. D 78, 123503 (2008) [arXiv:0808.3482 [gr-qc]]; F. Yu, J. Zhang, J. Lu, W. Wang and Y. Gui, Phys. Lett. B 688, 263 (2010) [arXiv:1004.2092 [astroph.CO]]; X. M. Chen, Y. G. Gong and E. N. Saridakis, JCAP 0904, 001 (2009) [arXiv:0812.1117 [gr-qc]].

[10] J. H. He and B. Wang, JCAP 0806, 010 (2008) [arXiv:0801.4233 [astro-ph]]; J. H. He, B. Wang and Y. P. Jing, JCAP 0907, 030 (2009) [arXiv:0902.0660 [gr- 
qc]]; J. H. He, B. Wang and P. Zhang, Phys. Rev. D 80, 063530 (2009) [arXiv:0906.0677 [gr-qc]]; J. H. He, B. Wang and E. Abdalla, arXiv:1012.3904 [astroph.CO]; C. G. Boehmer, G. Caldera-Cabral, R. Lazkoz and R. Maartens, Phys. Rev. D 78, 023505 (2008) [arXiv:0801.1565 [gr-qc]]; Z. K. Guo, N. Ohta and S. Tsujikawa, Phys. Rev. D 76, 023508 (2007) [arXiv:astroph/0702015]; J. Q. Xia, Phys. Rev. D 80, 103514 (2009) [arXiv:0911.4820 [astro-ph.CO]]; L. L. Honorez, B. A. Reid, O. Mena, L. Verde and R. Jimenez, JCAP 1009, 029 (2010) [arXiv:1006.0877 [astro-ph.CO]]; M. Baldi, arXiv:1005.2188 [astro-ph.CO].

[11] J. Valiviita, R. Maartens and E. Majerotto, Mon. Not. Roy. Astron. Soc. 402, 2355 (2010) [arXiv:0907.4987 [astro-ph.CO]].

[12] R. G. Cai and Q. Su, Phys. Rev. D 81, 103514 (2010) [arXiv:0912.1943 [astro-ph.CO]].

[13] M. Chevallier and D. Polarski, Int. J. Mod. Phys. D10, 213-224 (2001) [gr-qc/0009008]; E. V. Linder, Phys. Rev. Lett. 90, 091301 (2003) [astro-ph/0208512].

[14] R. Amanullah et al., Astrophys. J. 716, 712 (2010) [1004.1711 [astro-ph.CO]].

[15] S. Nesseris and L. Perivolaropoulos, Phys. Rev. D 72, 123519 (2005) [arXiv:astro-ph/0511040]; L. Perivolaropoulos, Phys. Rev. D 71, 063503 (2005) [arXiv:astro-ph/0412308]; S. Nesseris and L. Perivolaropoulos, JCAP 0702, 025 (2007) [arXiv:astro-ph/0612653].

[16] B. A. Reid et al. [SDSS Collaboration], Mon. Not. Roy. Astron. Soc. 401, 2148 (2010) [0907.1660 [astro-ph.CO]].

[17] D. J. Eisenstein, W. Hu, Astrophys. J. 496, 605 (1998) [astro-ph/9709112].

[18] E. Komatsu et al. [WMAP Collaboration], Astrophys. J. Suppl. 192, 18 (2011) [1001.4538 [astro-ph.CO]].
[19] W. Hu and N. Sugiyama, Astrophys. J. 471, 542 (1996) [astro-ph/9510117].

[20] J. R. Bond, G. Efstathiou and M. Tegmark, Mon. Not. Roy. Astron. Soc. 291, L33 (1997) [astro-ph/9702100].

[21] D. Stern, R. Jimenez, L. Verde, M. Kamionkowski and S. A. Stanford, JCAP 1002, 008 (2010) [arXiv:0907.3149 [astro-ph.CO]]; A. G. Riess et al., Astrophys. J. 699, 539 (2009) [arXiv:0905.0695 [astro-ph.CO]].

[22] E. Gaztanaga, A. Cabre and L. Hui, Mon. Not. Roy. Astron. Soc. 399, 1663 (2009) [arXiv:0807.3551 [astro$\mathrm{ph}]$.

[23] S. W. Allen, D. A. Rapetti, R. W. Schmidt, H. Ebeling, G. Morris and A. C. Fabian, Mon. Not. Roy. Astron. Soc. 383, 879 (2008) [arXiv:0706.0033 [astro-ph]].

[24] R. S. Goncalves, J. S. Alcaniz, A. Dev and D. Jain, arXiv:0910.4141 [astro-ph.CO].

[25] S. Basilakos, M. Plionis and J. Sola, Phys. Rev. D 80, 083511 (2009) [arXiv:0907.4555 [astro-ph.CO]]; I. L. Shapiro, J. Sola, C. Espana-Bonet and P. RuizLapuente, Phys. Lett. B 574, 149 (2003) [arXiv:astroph/0303306]; I. L. Shapiro, J. Sola and H. Stefancic, JCAP 0501, 012 (2005) [arXiv:hep-ph/0410095]; M. L. Tong and Y. Zhang, Phys. Rev. D 80, 023503 (2009) [arXiv:0906.3646 [gr-qc]]; Y. T. Wang, Y. X. Gui, L. X. Xu and J. B. Lu, Phys. Rev. D 81, 083514 (2010) [arXiv:1004.3341 [astro-ph.CO]].

[26] E. Elizalde, S. Nojiri, S. D. Odintsov and P. Wang, Phys. Rev. D 71, 103504 (2005) [arXiv:hep-th/0502082]. S. Nojiri and S. D. Odintsov, Phys. Lett. B 649, 440 (2007) [arXiv:hep-th/0702031].

[27] J. Z. Ma and X. Zhang, Phys. Lett. B 699, 233 (2011) [arXiv:1102.2671 [astro-ph.CO]]. 